\title{
O ENVELHECIMENTO, O CUIDADO COM O IDOSO E A DOENÇA DE ALZHEIMER
}

\author{
Brenda Bianca Andrade Sales Lourinho ${ }^{1}$ e Wilson Fernandes Ramos ${ }^{2}$
}

1. Enfermeira, Especialista em Unidade de terapia intensiva, Escola Superior da Amazônia/ESAMAZ, Belém, PA. (brenda.bianca@hotmail.com)

2. Engenheiro Agrônomo, Doutorando em Ciências Ambientais, Universidade Federal do Pará/UFPA, Belém, PA, Brasil. (wilson.f.ramos18@hotmail.com)

Recebido em: 04/10/2019 - Aprovado em: 30/11/2019 - Publicado em: 15/12/2019 DOI: 10.18677/EnciBio_2019B41

\begin{abstract}
RESUMO
O envelhecimento faz parte da realidade da maioria das sociedades, pois a pirâmide etária sofre uma mudança gradativa. $O$ envelhecimento faz com que o ser humano passe por inúmeras alterações no campo biológico e psicológico podendo acarretar o surgimento de doenças crônicas degenerativas como a Doença de ALZHEIMER. Assim, o objetivo deste trabalho é discutir a partir de trabalhos publicados a temática do envelhecimento, os cuidados com o idoso e a Doença de Alzheimer, que está relacionada com a idade avançada, focando, principalmente neste tipo de demência que é bastante estudada, porém sem cura. Para tal, foi utilizada a revisão da literatura especializada que é aquela elaborada a partir de material já publicado e documental. Dessa forma, Devido ao envelhecimento doenças de cunho psicológico acabam surgindo e causando preocupação de forma geral, entre essas doenças está a de ALZHEIMER. Entretanto, a revisão mostrou que o cuidado com o idoso portador dessa doença, pela família e profissionais capacitados diminui essa problemática. Assim, o envelhecimento é algo natural que pode ser saudável ou não, que para este caso, o cuidado e o respeito para com o idoso é fundamental, sendo o papel da família importantíssimo neste processo. Inclui-se nesta relação o cuidador, que pode ou não ser um familiar. Este profissional é de indiscutível importância, pois é ele que ficará grande parte do tempo na companhia e realizando os cuidados com o idoso, principalmente, aquele que é portador de alguma demência como a Doença de Alzheimer.
\end{abstract}

PALAVRA-CHAVE: Cuidador, doença de Alzheimer, envelhecimento.

\section{AGING, CARING FOR ELDERLY AND ALZHEIMER'S DISEASE}

\begin{abstract}
ABASTRACT
Aging is part of the reality of most societies, as the age pyramid undergoes a gradual change. Aging causes the human being to undergo numerous changes in the biological and psychological field and may lead to the emergence of degenerative chronic diseases such as ALZHEIMER Disease. Thus, the objective of this paper is to discuss from published papers the theme of aging, care with the elderly and Alzheimer's disease, which is very related to advanced age, focusing mainly on this type of dementia that is widely studied, but without cure. For this, we used the review
\end{abstract}


of specialized literature that is the one elaborated from already published and documentary material. Thus, Due to aging psychological illnesses end up arising and causing concern in general, among these diseases is that of ALZHEIMER. However, the review showed that care for the elderly with this disease, family and trained professionals reduces this problem. Thus, aging is something natural that can be healthy or not, which in this case, care and respect for the elderly is essential, and the role of the family is very important in this process. This relationship includes the caregiver, who may or may not be a family member. This professional is of unquestionable importance, since he is the one who will spend most of his time in the company and performing care with the elderly, especially those with dementia such as Alzheimer's disease.

KEYWORDS: Aging, Alzheimer's disease, career

\section{INTRODUÇÃO}

O envelhecimento faz parte da realidade da maioria das sociedades, principalmente as desenvolvidas, pois claramente a pirâmide etária está sofrendo uma mudança gradativa. Dessa forma, verifica-se que o número de idosos só aumenta, mormente, quando comparado com as taxas de natalidade. Diante disso, estima-se que para o ano de 2050 haverá cerca de dois bilhões de pessoas com 60 anos ou mais no mundo, a maioria vivendo em países em desenvolvimento (MOREIRA et al., 2018).

A mudança de alguns indicadores como a queda da fecundidade e da mortalidade e o aumento da expectativa de vida acarreta no envelhecimento populacional que não é homogêneo para todos os seres humanos, sofrendo influência dos processos de discriminação e exclusão associados ao gênero, à etnia, ao racismo, às condições sociais e econômicas, à região geográfica de origem e à localização de moradia. O envelhecimento não é isonômico em todas as regiões do globo (MIRANDA et al., 2016).

Dessa forma, para Moreira, et al., (2018) o envelhecimento é o processo natural, de diminuição progressiva da reserva funcional dos indivíduos que em condições normais, não costuma provocar qualquer problema. No entanto, em condições de sobrecarga como doenças, acidentes e estresse emocional, pode levar a uma condição patológica que requeira assistência. Destaca-se que certas alterações decorrentes do processo de envelhecimento podem ter seus efeitos minimizados pela assimilação de um estilo de vida mais ativo.

O envelhecimento faz com que o ser humano passe por inúmeras alterações no campo biológico e psicológico que somadas ao meio em que ele vive, ou ao modo de vida do indivíduo, podem estar relacionado ao surgimento ou não de doenças crônicas degenerativas, que podem acarretar em transtornos mentais, como demências, transtornos psicóticos, depressão e ansiedade. Essas demências consistem em uma síndrome cujo efeito é acometer o funcionamento cerebral e podem ter natureza crônica e progressiva (BURLA et al., 2012).

Em relação as demências a causa mais comum é a Doença de Alzheimer, sendo uma das principais causas de incapacidade na velhice, demandando cuidados durante todo o curso desta enfermidade que culmina da dependência total da pessoa doente. A Doença de Alzheimer é um tipo de demência de caráter crônico, degenerativo, progressivo e irreversível que pode causar diversos sintomas, como perda de memória, mudanças comportamentais, afasia, delírios e alucinações (BURLA et al., 2012). Com isso, o objetivo deste trabalho é discutir a partir de trabalhos publicados a temática do envelhecimento, os cuidados com o idoso e a 
Doença de Alzheimer, que está muito relacionada com a idade avançada, focando, principalmente neste tipo de demência que é bastante estudada, porém sem cura.

\section{MATERIAL E MÉTODOS}

O presente estudo se constitui em uma revisão da literatura especializada, que pode ser classificado, quanto aos procedimentos técnicos, como uma pesquisa bibliográfica, visto que é aquela elaborada a partir de material já publicado e documental, a qual é feita a partir de documentos que não sofreram uma análise crítica de especialista ou não foram publicados em revistas cientificas (GIL, 2010).

Assim, segundo Cooper (1989), esse tipo de pesquisa se caracteriza por ser um método que agrega os resultados obtidos de pesquisas primárias sobre a mesma temática, objetivando, com isso, sintetizar e analisar esses dados para desenvolver uma explicação mais abrangente de um fenômeno específico, que neste caso é a problemática do envelhecimento e suas implicações.

A presente revisão foi construída entre fevereiro e junho de 2019, em que se realizou uma consulta a livros, periódicos e artigos científicos, os quais foram selecionados através de busca no banco de dados do Scielo, periódicos CAPES e da Bireme, a partir das fontes Medline e Lilacs.

Para realização da busca nos bancos de dados, foram utilizadas terminologias cadastradas nos Descritores em Ciências da Saúde criados pela Biblioteca Virtual em Saúde desenvolvido a partir do Medical Subject Headings da U.S. National Library of Medicine, que permite o uso da terminologia comum em português, inglês e espanhol. As palavras-chave utilizadas na busca foram envelhecimento, doenças relacionadas ao envelhecimento, o idoso e a doença de Alzheimer, entre outros. Foram excluídos outros estudos que não tinham como foco doenças relacionadas ao envelhecimento.

Justifica-se a escolha da revisão da literatura através da definição como sendo uma aplicação de estratégias científicas que limitam o viés da seleção de artigos, onde se avalia criticamente os artigos e se sintetizam todos os estudos relevantes em uma temática específica (PERISSÉ, 2001).

Dessa forma, de acordo com Mancini (2007) a importância desse tipo de estudo é que pode-se criar uma forte base de conhecimentos, capaz de guiar a prática profissional e identificar a necessidade de novas pesquisas. Ademais, Hek (2000), destaca que as revisões de literatura se constituem em um método moderno para a avaliação simultânea de um conjunto de dados.

\section{ENVELHECIMENTO NA ATUALIDADE E ALZHEIMER}

Historicamente, em muitas sociedades, discute-se sobre a longevidade e o prolongamento da vida, que para muitas pessoas é motivo de estudos. Segundo Veras (2009), o envelhecimento só pode ser considerado como uma real conquista na medida em que se agregue qualidade aos anos adicionais de vida. Sobre qualidade de vida na velhice destaca-se a inserção na sociedade, a sua função e importância no seio familiar e muitas vezes a questão de empregabilidade.

Segundo Capucha, (2017), o processo de envelhecimento normalmente está associado a um bom nível de saúde, com o avanço da medicina, em muitos países, a expectativa de vida ultrapassa os 70 anos. Dessa forma, em quase todo o mundo, o contingente que mais cresce é o de pessoas com idade igual ou superior a 60 anos. Segundo Burla et al., (2012), as projeções demográficas apontam para 2020 um contingente de 29,8 milhões de pessoas com 60 anos ou mais e de 4,7 milhões 
acima de 80 anos, significando, respectivamente, um acréscimo de 9,2 milhões e 1,7 milhões de indivíduos na faixa etária de 80 anos ou mais, comparado ao contingente de 2010.

Devido a mudança da pirâmide etária, a complexidade envolvendo os problemas sociais relacionados ao aumento da expectativa de vida reflete diretamente na saúde do idoso que está diretamente relacionada com a sua funcionalidade global (MORAES, et al., 2010). Assim, viver mais implica, naturalmente, no declínio fisiológico das funções orgânicas, com isso a probabilidade do surgimento de doenças crônicas como as demências são altíssimas, embora não seja um componente normal de envelhecimento (BURLA et al., 2012).

As demências consistem em uma síndrome cujo efeito é acometer o funcionamento cerebral, de natureza crônica e progressiva, comprometendo várias funções cerebrais, incluindo memória, raciocínio, orientação, compreensão, cálculo, capacidade de aprendizagem, linguagem e julgamento (BURLA et al., 2012). Para estes autores, a causa mais comum da demência é a Doença de Alzheimer, sendo uma das principais causas de incapacidade na velhice.

A Doença de Alzheimer é um dos desafios sociais e de saúde que são mais importantes nas sociedades industrializadas, com o aumento da expectativa de vida e o progressivo envelhecimento ocorre o desenvolvimento de condições associadas ao envelhecimento (MOREIRA, et al., 2018). Em 1906, a doença de Alzheimer foi diagnosticada pelo médico Alois Alzheimer, no trigésimo sétimo congresso do sudoeste Alemão de Psiquiatria, em Tribigem. Neste congresso o Médico Alois expôs o caso de sua paciente, Sra. Auguste D., 50 anos, que apresentava um tipo de demência com progressiva perda cognitiva. Então, após sua morte, feito a necropsia, foi observada a presença de placas e emaranhados neurofibrilares e algumas alterações ateroscleróticas, denominando este quadro clinico posteriormente de Doença de Alzheimer (CECATO, et al., 2018).

Para Jerônimo (2016), tecnicamente a doença do tipo Alzheimer é definida pelo CID-10 (Código Internacional de doenças) como uma doença cerebral degenerativa primária de etiologia desconhecida, com aspectos neuropatológicas e neuroquímicos característicos, sendo um tipo de doença de caráter crônico, degenerativo, progressivo e irreversível. Usualmente é de natureza crônica ou progressiva, na qual há perturbação de múltiplas funções corticais superiores, incluindo memória, pensamento, orientação, compreensão, cálculo, capacidade de aprendizagem, linguagem e julgamento.

De acordo com Siqueira et al. (2019), a Doença de Alzheimer tem início de modo insidioso e se desenvolve por vários anos lentamente. As alterações neuropatológicas e bioquímicas podem ser divididas em duas áreas gerais: mudanças estruturais que incluem os enovelados neurofibrilares e alterações nos neurotransmissores ou sistemas neurotransmissores que estão ligadas as mudanças patológicas que ocorrem no decorrer da doença.

A perda neural e a degeneração sináptica ocorrem devido dois tipos de lesões existentes na doença as placas senis e os emaranhados neurofibrilares. As placas senis são estruturas extracelulares constituídas de uma proteína, a betaamiloide, e origina-se na proteína precursora do amiloide, existente em praticamente todas as células humanas, mas sem funções bem delineadas, sabe-se, no entanto que esta age como inibidora de proteases (BERNARDO; RAYMUNDO, 2018).

É comum que os sinais iniciais da Doença de Alzheimer sejam confundidos com o processo natural do envelhecimento, com isso a busca por orientação 
profissional tende a ser adiada e consequentemente a doença é diagnosticada tardiamente, a certeza do diagnóstico só pode ser obtida por meio do exame microscópico do tecido cerebral dos doentes após o falecimento. Na prática médica o diagnóstico é clinico, depende da avaliação feita pelo médico associado a exame de sangue, de imagem e do próprio histórico do paciente (ABRAZ, 2010). Dessa forma, o diagnóstico da doença de Alzheimer é de exclusão, porém o definitivo depende do exame histopatológico do cérebro post-mortem (BARROS, et al., 2018).

Havendo o diagnóstico, o tratamento será multidisciplinar, envolvendo todas as peculiaridades relacionadas aos sinais e sintomas, com o objetivo de estabilizar o comprometimento cognitivo, o comportamento e a prática das atividades diárias. Os fármacos utilizados são os colinérgicos: Donepezila, Galantamina e Rivastigmina tanto para a doença de Alzheimer leve quanto a moderada. Estes medicamentos apresentam propriedades farmacológicas levemente diferentes, porém ambos agem inibindo a degradação da molécula de acetilcolina, o neurotransmissor associado à função de memória, por bloquear a enzima acetilcolinesterase (JESUS et al., 2019).

Apesar de não haver cura, o tratamento vai desde o uso de fármacos que aliviam os sintomas até o acompanhamento multiprofissional, que prestará de forma integral uma assistência ao portador da Doença de Alzheimer e, consequentemente, aos seus cuidadores.

\section{O CUIDADOR, O IDOSO COM ALZHEIMER E A EDUCAÇÃO EM SAÚDE}

O crescimento do número de idosos que necessitam de tratamento domiciliar e o cuidado envolvendo familiares, parentes e amigos só vem crescendo nos últimos tempos. Verifica-se que é uma área crescente para o mercado de trabalho, pois o número de idosos cresce, exigindo um número considerado de profissionais e com boa capacitação.

Assim, segundo Brum et.al. (2013):

A necessidade de um cuidador continuo representa uma demanda para a família em que o cuidado é visto como algo natural que deva ser realizado pela mulher, entretanto devido as famílias estarem se tornando cada vez menor, mas com um número expressivo de idosos, os problemas decorrentes do envelhecimento se estabelecem.

De acordo com o exposto, é nesse contexto que o acompanhamento de um idoso com Alzheimer requer maior suporte e a necessidade de uma assistência adequada. Para tal suporte e assistência é preciso de um profissional adequado e treinado. Assim, foi expedida a Portaria no 2528 de 2006, que aprova a Política Nacional de Saúde da Pessoa Idosa, nesta portaria o cuidador é conceituado da seguinte forma:

Toda pessoa, membro ou não da família, que, com ou sem remuneração, formal ou informal, presta cuidados ao idoso que depende de auxilio em suas atividades diárias, como: alimentação, higiene pessoal, medicação, companhia aos serviços de saúde, serviços de bancos ou farmácias, entre outros (BRASIL, 2006).

Neste contexto, em grande parte dos casos o primeiro cuidador é alguém da família, ou seja, os filhos, netos ou irmãos, que não apresentam um treinamento adequado e fazem puramente por amor. Na maioria das vezes o cuidador além de 
prestar cuidados ao idoso, realiza tarefas domésticas, bem como há fatores que atuam como estressores, "é o fato da doença incapacitante do idoso requerer cuidados básicos contínuos da vida e a incidência de doença na própria família do cuidador, dificultando a tarefa do cuidar" (OLIVEIRA; D'ELBOUX, 2012).

Nestas situações, com a presença de muitos fatores estressores, os cuidadores deixam de lado a profissão, atividade de lazer, o autocuidado, o que pode levar a diminuição da qualidade de vida refletindo no seu trabalho e no cuidado prestado ao idoso (NASCIMENTO, et al., 2018). Pelas características incapacitantes da doença de Alzheimer, a demanda por cuidados e supervisão constante aumenta, este papel de cuidar é na maioria das vezes desempenhado por um membro familiar trazendo modificações na qualidade de vida e na capacidade funcional dos indivíduos. Segundo o Estatuto do Idoso, Lei $\mathrm{n}^{\circ}$ 10.741, de 1ํㅡㄹ de outubro de 2003:

Art. 3ํ É obrigação da família, da comunidade, da sociedade e do poder político assegurar ao idoso, com absoluta prioridade, a efetivação o direito à vida, à saúde, à alimentação, à educação, à cultura, ao esporte, ao lazer, ao trabalho, à cidadania, à liberdade, à dignidade, ao respeito e a convivência familiar e comunitária (BRASIL, 2003)

No momento em que a família assume o papel de cuidador do idoso com Doença de Alzheimer, há uma mudança significativa na rotina social, financeira, emocional e familiar. A importância de ser um familiar o atuante no cuidado do idoso é que a relação entre o cuidador e os idosos é tão íntimo e próximo que o próprio cuidador modifica o seu modo de viver em prol do cuidado ao idoso, bem como a questão de afeto. Essa mudança nem sempre é realizada pela vontade do cuidador, mas por conjuntura, porém pode ser amenizado os problemas quando a relação envolve a participação, o cuidado, a presença na vida do idoso por amor e fidelidade, doando-se para o outro, estando presente (SEIMA, et al., 2014)

Dessa forma, entende-se que 0 ato de cuidar é uma tarefa que envolve múltiplos sentimentos, claramente a questão de ser alguém da família ajuda, é preciso ter amor e ser paciente para que a escolha pela doação, dedicação e abdicação seja incondicional, mesmo sabendo que a escolha por vivenciar o cuidado junto ao idoso com Alzheimer não terá possibilidade de reconhecimento e retorno afetivo por parte do portador (NASCIMENTO, et al., 2018). Além disso, é necessário ter recursos financeiros a fim de facilitar a aquisição de tecnologia assistivas para melhorar a condição de saúde e de vida tanto dos cuidadores quanto do idoso.

A fragilidade referente à carência de suporte ao cuidador reflete na sobre carga deste, em uma rotina desgastante, ao automatismo do cotidiano gerando ambivalência de sentimentos que se não interpretados de forma correta poderá influir negativamente na assistência ao cuidado deste idoso (SEIMA, et al., 2014). Neste caso, o treinamento e a educação para o cuidado com o idoso portador dessa demência é fundamental.

Assim, a prática relacionada à Educação e Saúde no campo da saúde merece atenção e um maior incentivo, principalmente quando relacionado ao envelhecimento (TEIXEIRA; MOTA, 2011). A educação em saúde tem por objetivo permitir que as pessoas de uma comunidade aprendam a interagir de forma participativa com o sistema de saúde e desempenhem o seu papel de forma individual e coletiva, na promoção, manutenção e restauração da saúde (ARAUJO et al., 2013). Sobre a realização da Educação em Saúde, e a sua importância no contexto familiar e social Araújo et al. (2013) defende que: 


\begin{abstract}
A efetivação da educação em saúde dispõe de uma base estruturalmente sólida para proporcionar o bem estar individual e da coletividade. O ensino é um instrumento que todos os profissionais de saúde podem e devem utilizar para cuidar dos seus pacientes, para promover a saúde e prevenir doenças, englobando, nesse contexto, a família e os cuidadores, a fim de embutir nos hábitos já pré-estabelecidos o desenvolvimento de comportamentos de saúde efetivos e modificar os padrões de estilo de vida predispostos ao risco de saúde.
\end{abstract}

A partir disso pode-se perceber a importância de dar ênfase as ações relacionadas a Educação em Saúde, no contexto familiar e social, e da utilização da comunicação como instrumento terapêutico e promotor de possibilitar o acesso à informação e a troca de experiências que contribuem para prevenção de morbidades e promoção da saúde, que acabam por estimularem espaços de humanização, inclusão social e participação do cidadão (PEREIRA et al., 2012).

A prática da educação em saúde tem como finalidade não somente de propagação de conhecimentos, mas também a adoção de práticas educativas que busquem a autonomia dos sujeitos e desenvolvam o senso de responsabilidade, tanto por sua própria saúde, como pela saúde da coletividade (ARAUJO et al., 2013). Vale ressaltar sobre a educação em saúde que quando esta é voltada para uma demanda necessitada, torna-se uma aliada no processo de trabalho, pois consegue direcionar-se aos problemas comuns da comunidade, a exemplo da população idosa que cresce a cada dia e precisa de uma atenção diferenciada, tendo como finalidade de suprir as necessidades relacionadas a saúde dessa população.

A promoção da saúde está associada a ampla concepção do processo saúde-doença que juntos essas duas ideias passam a articular os saberes técnicos e populares, além de mobilizar recursos públicos e privado em diversos sentidos, para o enfrentamento dos problemas de saúde e seus determinantes, tendo a educação em saúde como importante componente neste sistema (ARAUJO et al., 2013). E, para Dias júnior; Costa (2016):

A prática da educação em saúde como um caminho integrador do cuidar constitui um espaço de reflexão-ação, fundado em saberes técnicocientíficos e populares, culturalmente significativos para o exercício democrático, capaz de provocar mudanças individuais e prontidão para atuar na família e na comunidade, interferindo no controle e na implementação de políticas públicas, contribuindo para a transformação social.

Nesse sentido é irrefutável o papel do enfermeiro como educador em saúde, seja na sua versão individual ou coletiva (DIAS JÚNIOR; COSTA, 2016). Observa-se que o ensino da Educação em Saúde na enfermagem se inicia ainda na formação e, porém essa realidade muitas vezes não ocorre nas instituições de ensino superior, que não oferecem uma saída para esse problema existente entre teoria e prática. Portanto reflete-se sobre a necessidade de implantação de um processo educativo que aborda esse conteúdo especifico, relacionado a educação em saúde, e que defenda o uso de tecnologias educativas pelos profissionais da saúde, com a finalidade de implantar na ideia da formação da enfermagem, um trabalho participativo, democrático e cidadã com os distintos grupos da população (TEIXEIRA; MOTA 2011). 
Para Pereira et al., (2012), na enfermagem, a tecnologia gera impacto significativo no processo de trabalho, sendo designada como a aplicação dos conhecimentos científicos de modo sistemático no auxílio para melhor atender o ser humano. Observa-se que a enfermagem depara-se constantemente com o desafio de acompanhar o desenvolvimento tecnológico sem deslembrar os aspectos éticos e humanitários que devem ser inerentes à profissão.

\section{OS SERVIÇOS DE APOIO OFERECIDOS A IDOSOS COM ALZHEIMER (ABRAZ)}

Devido a preocupação em relação ao envelhecimento e por conseguinte a aumento nos casos de Alzheimer, foi fundada em 16 de agosto de 1991 a Associação Brasileira de Alzheimer (ABRAZ) que reúne familiares, familiarescuidadores e cuidadores profissionais em seu quadro associativo, com a finalidade de, a partir de suas vivências e conhecimentos, desenvolver ações em favor das pessoas acometidas pela Doença de Alzheimer (DA) e oferecer apoio ao familiarcuidador (ABRAZ, 2015).

A ABRAZ é uma entidade privada de natureza civil, sem fins lucrativos, que tem como missão ser o núcleo central, em todo o País, das pessoas envolvidas com a Doença de Alzheimer e outras demências. Sediada em São Paulo, a ABRAz possui, atualmente, cerca de 13 mil associados e atua nos Estados por meio das Regionais (JESKE et al. 2018). O trabalho é desenvolvido por voluntários, profissionais das áreas de saúde, educação, jurídica e outros, e por familiares de pessoas com a Doença de Alzheimer.

Esta Associação oferece apoio e contribui para a superação de dificuldades enfrentadas desde o momento do diagnóstico até as etapas mais avançadas da doença. Muitos dos familiares que se beneficiam do trabalho oferecido tornam-se voluntários e multiplicadores dos conhecimentos e das experiências adquiridas (ABRAZ, 2015).

Uma das assistências é o Grupo de Apoio que é um espaço de encontro, aprendizagem e troca de experiências oferecido especialmente para familiares e cuidadores de idosos com Doença de Alzheimer, no qual os participantes têm a oportunidade de refletir sobre a tarefa de cuidado sob novas perspectivas, ao encontrar novas estratégias para superar dificuldades e descobrir novas formas de lidar com o cotidiano modificado. Oferece também grupos de estimulação e de convívio social para idosos saudáveis e com quadros demenciais (SALES, 2018).

Os principais benefícios que os grupos de apoio oferecem para os cuidadores são 0 acesso as informações sobre a doença e os tratamentos, melhorando com isso a tomada de decisões e consequentemente a qualidade do cuidado. Sobre a aceitação da nova situação, criaram o desenvolvimento de um enfrentamento mais positivo e saudável em relação ao adoecimento e perdas associadas visando a qualidade de vida de todos os envolvidos tanto o idoso quanto o cuidador estimulando a interação com o idoso e uma maior compreensão das necessidades desse idoso com a doença de Alzheimer, suas estratégias de manejo e sintomas (ABRAZ, 2015).

Os grupos de Apoio da ABRAZ estão divididos em dois que são os Informativos e de Apoio Social e Emocional. Nos Grupos Informativos, as informações acerca da doença de Alzheimer são passadas aos cuidadores familiares ou não através de palestras conferidas por convidados especialistas e pelo coordenador do grupo de apoio. Abordam informações sobre a doença em si, os tratamentos, cuidados necessários a esse idoso com demência, entre outros, 
sendo que o cuidador pode também participar de forma ativa da palestra, contando suas experiências e frustrações, com a finalidade de envolver os participantes do debate com o tema em questão que e a doença de Alzheimer e contribuir também com o palestrante (ABRAZ, 2015).

\begin{abstract}
Esta modalidade de grupo auxilia o cuidador a entender sobre a Doença de Alzheimer e a identificar, bem como lidar, com sintomas e dificuldades dos pacientes no convívio cotidiano. Trata-se de um espaço voltado para a aprendizagem de informações que contribuam para preparar os cuidadores para a tarefa de cuidado, tornando-os mais seguros e confiantes para uma relação de qualidade e com atenção às necessidades do idoso vulnerável. As aulas ministradas permitem esclarecer dúvidas e ideias erradas sobre a doença, sua evolução e tratamentos (farmacológicos e não farmacológicos) (ABRAZ, 2015).
\end{abstract}

Sobre os Grupos de Apoio Social e Emocional, estes se caracterizam essencialmente como um espaço de troca de experiência e reflexão, para que o cuidador possa estar diante de pessoas que passam por situações semelhantes, experimentando sensação de pertencimento, acolhimento e conforto.

\begin{abstract}
Nos grupos há espaço para o cuidador interagir e compartilhar dificuldades práticas do dia a dia e aspectos emocionais envolvidos no cuidado do idoso com doença neurodegenerativa. Trata-se, portanto, de um ambiente que favorece a convivência e no qual há a possibilidade de os participantes relatarem suas histórias e discutirem suas principais dificuldades para a busca coletiva de alternativas e estratégias eficientes e seguras que minimizem sofrimento e garantam investimento em qualidade de vida. Assim, esses encontros favorecem que o cuidador tenha mais recursos para lidar com o idoso, receba apoio e se organize melhor, utilize recursos pessoais para encontrar soluções para os problemas, expresse sentimentos e vivências particulares e atribua novo sentido às suas experiências ao encontrar novas formas de enfrentamento (ABRAZ, 2015).
\end{abstract}

A Associação Brasileira de Alzheimer (ABRAZ) orienta quanto ao cuidado ao doente de Alzheimer, com dicas de como cuidar e lidar com esse idoso e os direitos desses. Os temas discutidos envolvem situações de dificuldades na rotina de cuidado (dar banho, oferecer medicamento), mudanças na vida pessoal e familiar após o adoecimento, necessidade de tomar decisões, problemas para lidar com alterações de cognição e comportamento do idoso com demência (sintomas da Doença de Alzheimer) (ABRAZ, 2015).

A ABRAZ também promove orientações aos cuidados com o cuidadorfamiliar, sobre o impacto emocional, já que estes participam do processo de degeneração cognitiva geradora de incapacitação, que gera no familiar-cuidador o medo em relação ao futuro do paciente e de si mesmo, assistir ao seu familiar perder gradativamente sua identidade gera intenso sofrimento e impotência, e o relacionamento com o paciente passa a ser um confronto com múltiplas e cumulativas perdas que precisam ser constantemente adaptadas, e sobre a importância da revelação do diagnóstico, pois devido ao comprometimento cognitivo é essencial que, caso a família opte por contar ao paciente sobre seu diagnóstico, a revelação seja feita no estágio inicial da doença para favorecer a compreensão e o registro dessa informação (ABRAZ, 2015).

Os Grupos voltados para idosos saudáveis visam a exercitar as funções cognitivas para a manutenção de seu bom funcionamento. A proposta baseia-se na 
premissa de que quanto mais utilizado é o cérebro, melhor prognóstico a pessoa teria se desenvolvesse a Doença de Alzheimer (DA). Os encontros envolvem momentos prazerosos de interação e alcance de desafios mentais. Dentre as atividades oferecidas, destacam-se as de estimulação cognitiva, como oficinas de memória e desafios mentais, aulas de informática, palestras, artesanato e exibição de filmes (ABRAZ, 2015).

Os Grupos voltados para idosos com demência promovem interação social e atividades que favorecem 0 estímulo das funções cognitivas. Os pacientes participam de atividades variadas que abordam diferentes áreas. Também são oferecidas atividades lúdicas, envolvendo jogos, exercícios de atenção, exercícios com palavras, construções, música, dança e tarefas que permitam reflexão, decisão e satisfação (JESKE et al. 2018).

Outro compromisso da ABRAZ é o de informar a população sobre o Alzheimer, com a finalidade de favorecer o diagnóstico precoce. Além dos grupos de apoio, são realizadas campanhas, palestras, cursos e debates abertos à população, a fim de esclarecer sobre a Doença de Alzheimer, os sintomas e tratamentos. A ABRAz atualmente promove ações informativas anuais e mensais, como o Mês mundial da Doença de Alzheimer, Cine ABRAZ: com filmes e debates acerca do assunto, além de ações em comunidades e instituições com programa psicoeducativo (ABRAZ, 2015).

\section{A ENFERMAGEM, FAMÍLIA E O IDOSO COM ALZHEIMER}

O aumento da população idosa é um fenômeno mundial, principalmente nos países em desenvolvimento, em que as taxas de natalidade e mortalidade são baixíssimas, levando a uma mudança na estrutura da pirâmide etária. Esse aumento leva, por consequência, a um avanço de doenças crônico-degenerativas, que logo, acarretam um aumento dos gastos permanentes por parte da família cuidadora (MEDEIROS, 2016), bem como de investimentos do Estado na saúde do idoso e em profissionais capacitados.

Em relação a saúde provida pelo estado, um dos maiores desafios é orientar a família e ou o cuidador desse idoso dando um maior esclarecimento e treinamento sobre essas demências, além de realizar a promoção integral da saúde. Dessa forma, um profissional que está próximo e que tem um papel fundamental no tratamento e na saúde do idoso é o enfermeiro que é um propagador dessas informações acerca das características de cada uma delas, e os procedimentos adequados ao conjunto de ações que constituem o cuidado, tudo isso de acordo com a realidade cotidiana (OLIVEIRA et al., 2018). Diante disso, Brito, et al., (2017) comentam que:

\footnotetext{
A tendência atual é o aparecimento crescente de pessoas idosas que, apesar de viverem mais, apresentam uma associação de patologias crónicas. Pela evidência empírica sabe-se que o processo de envelhecimento, habitualmente, arrasta consigo doença e incapacidade, e que as doenças crónicas estão diretamente relacionadas com incapacidade funcional.
}

Geralmente é sobre a família que recai a responsabilidade principal pelo cuidado para com as pessoas idosas. Não há políticas que capacite uma pessoa ou um grupo de pessoas para prestar o cuidar adequado para atender as necessidades do idoso. Muitas vezes esse despreparo acaba tornando os familiares ou cuidadores 
informais mais vulneráveis ao adoecimento físico ou psíquico. Os profissionais de saúde, sobretudo os enfermeiros, tem um papel importante no desenvolvimento dessa capacitação (MEDEIROS et al., 2016).

Para Cavalcanti et al, 2018 a enfermagem observa a necessidade de prestar assistência de qualidade a esse cuidador-familiar e consequentemente a esse idoso, com isso faz-se necessário o acesso desse familiar-cuidador as informações referentes a patologia. Assim, a assistência de enfermagem é a principal fonte de auxílio para este familiar, para que este esteja preparado para enfrentar essas mudanças de forma possível.

profissional de enfermagem tem o papel de orientar quanto as necessidades e as instruções sobre o cuidado, a doença em si, a prevenção de acidentes, realização da higiene, a alimentação, tomada de medicamentos e ao amor, carinho e paciência que devem ser dados a esse idoso, esse novo cuidador passará a prestar uma assistência de mais qualidade ao idoso doente. Cavalcanti et al., (2018) defendem que:

\begin{abstract}
Os profissionais de saúde, principalmente o profissional de enfermagem devido suas técnicas de cuidado, formam com este familiar um tripé proporcionando-Ihe um ponto de apoio e ensinando-lhe com lidar com quaisquer dificuldades. $O$ cuidar é um processo dinâmico que depende não só da interação entra cuidador e ser cuidado, mas também do respeito e de ações planejadas devidamente a partir do conhecimento da realidade. $O$ familiar-cuidador só saberá como agir em determinadas situações se para isso este estiver preparado através de orientações dada a ele pelo profissional de enfermagem e outros.
\end{abstract}

A enfermagem fundamenta o conhecimento baseando-se no método científico para orientar a prática dos profissionais de enfermagem, facilitando a sistematização do processo de cuidar; portanto, existem modelos teóricos que auxiliam a sistematização de cuidar clinicamente em enfermagem, como o Modelo de Adaptação de Callista Roy (MAR), que é o fundamento para explicar o cuidado direto ao cuidador familiar e indireto ao idoso com Alzheimer (SENNA DA CRUZ, 2017).

Este modelo trabalha com cinco elementos essenciais no seu modelo de adaptação. $O$ primeiro fala da Pessoa que recebe o Cuidado de Enfermagem, o segundo sobre as Metas de Enfermagem que tem por finalidade promover respostas adaptativas em relação aos modos adaptativos, o terceiro fala sobre as atividades de Enfermagem que envolve a manipulação dos estímulos de modo a promover reações adaptativas. O quarto elemento é a Saúde que é o processo de tornar-se integrado e capaz de alcançar as metas de sobrevivência, crescimento, reprodução e controle. Já o quinto e último discute sobre o Ambiente, que são todas as condições, circunstâncias e influências que cercam e afetam o desenvolvimento e comportamento de pessoas e grupos ((SENNA DA CRUZ, 2017).

Para CARDOSO et al. (2018):

Conforme a utilização do Modelo de Adaptação de Callista Roy, que permite reconhecer as pessoas, mediante estímulos, podem se desencadear respostas, ora positivas, ora negativas, em situações estressantes. Compete, porém, à enfermeira atuar como mediador entre a objetividade técnica e a subjetividade humana, mediante estratégias para as ações do cuidar, capacitando as pessoas a desenvolverem mecanismos de enfrentamento destinados a diminuir as respostas negativas, favorecendo sua vivência e facilitando a realização do procedimento. 
Assim, a função do enfermeiro, nessas situações é promover a adaptação positiva do paciente e do cuidador, desenvolvendo ações relacionadas a avaliação e a intervenção. $\mathrm{Na}$ avaliação, o enfermeiro deve identificar as situações problemas e seus respectivos estímulos, já na intervenção, ele fará com que a pessoa se adapte, manipulando estes estímulos, de modo a eliminá-los (CESÁRIO et al., 2017).

Segundo os autores supracitados, a pessoa que recebe os cuidados de enfermagem é vista como um ser biopsicossocial, com mecanismos adaptativos inatos e adquiridos, os quais lhes permitem adaptar-se às mudanças que ocorrem, sejam internas ou externas. Vale dizer que o grau de adaptação é influenciado pelo desenvolvimento de sistemas de enfrentamento regulador que são os transmissores químicos, neurais e endócrinos e cogniscentes como os cerebrais superiores de percepção, julgamento e emoção. A constante interação das pessoas com seus ambientes está caracterizada por mudanças internas e externas nesse mundo em transformação e, para manterem sua própria integridade, adaptam-se continuamente a ele.

A utilização desta teoria da Adaptação de Callista Roy, permitiu reconhecer que as pessoas, mediante estímulos, podem desencadear respostas, ora positivas ora negativas, em situações estressantes, cabendo ao enfermeiro atuar como mediador entre a objetividade técnica e a subjetividade humana, elaborando estratégias para as ações do cuidar; capacitando as pessoas a criarem mecanismos de enfrentamento que possam diminuir as respostas negativas; favorecendo sua vivência e facilitando a realização do procedimento (CESÁRIO, 2017)

Observa-se a necessidade de adaptação desse cuidador a essa nova realidade. Nessa teoria tem-se o conceito de adaptação como eixo orientador da prática da enfermagem, que considera a pessoa como um sistema aberto, continuamente mudando e tentando se adaptar à realidade (OLIVEIRA et al., 2018).

\begin{abstract}
A pessoa, a família, a comunidade ou a sociedade são considerados, neste modelo, os receptores dos cuidados de enfermagem sendo, cada um deles, um sistema adaptativo holístico. Assim, o alvo dos cuidados de enfermagem, enquanto sistema aberto, está em constante interação com o ambiente, promovendo e submetendo-se a trocas de informação, matéria e energia (CAMPOS, et al., 2019).
\end{abstract}

Para Senna da cruz (2017), a Enfermagem como uma profissão dos cuidados de saúde que enfatiza a promoção à saúde, centraliza-se nos processos de vida humanos, nos grupos e na sociedade como um todo, sendo que a ciência e a prática expandem a capacidade de adaptação e melhora a transformação ambiental da pessoa. A partir disso enfatiza-se a necessidade que os profissionais de saúde têm, especialmente enfermeiros, de realizarem uma abordagem assistencial a família desse idoso e ao processo de envelhecimento em si. O Enfermeiro, ao se deparar com a família do idoso, deve promover o cuidar com orientações direcionadas, observando as suas especificidades e os ajudando no entendimento do autocuidado de quem cuida com respeito aos diversos aspectos que envolvem o ser humano (MEDEIROS, et al., 2016).

Apesar de já se prestarem alguns cuidados a grupos familiares, os desafios colocados à enfermagem incluem a família como unidade de cuidados, como sistema ou família como um contexto de cuidado. Esta alteração de paradigma exige uma avaliação personalizada para uma intervenção adequada à singularidade de cada família (BRITO, CAMARGO e CASTRO, 2017). 
Senna da cruz (2017), debate que nesse sentido os profissionais de enfermagem devem atentar-se também para o fator cultural que representa a singularidade de cada família. Dessa forma, o cuidado desponta como revelador do comportamento, a partir de uma complexa rede de interações, que abarca diferentes formas de conhecimento, valores, crenças e significados que moldam as ações de cuidado em um contexto sociocultural. Portanto, este estudo contribui com a Enfermagem no fomento de discussões acerca do cuidado que vêm sendo prestados pelos familiares aos idosos com doença de Alzheimer, uma vez que a obtenção de dados sobre o cuidado familiar do idoso é um fator fundamental para dinamizar medidas adequadas a essa população que permitam entender melhor a problemática vivenciada pelas famílias e, assim, encontrar subsídios para efetivas orientações.

Observa-se normalmente que a doença de uma pessoa afeta todos os membros do grupo familiar. Quando um dos membros de uma família é um dependente, isso requer por parte do corpo de enfermagem uma avaliação familiar, afim de reconhecer 0 impacto da doença naquele sistema familiar e o quanto 0 indivíduo depende daquela família, com o intuito de identificar as forças e as fragilidades dessa, para determinar o nível de apoio que esses idosos necessitam. Com isso se faz necessário que os profissionais visualizem esta realidade e prestem cuidados humanizados, holísticos ao idoso e à sua família (BRITO, et al., 2017).

\section{CONSIDERAÇÕES FINAIS}

Verificou-se com este trabalho que claramente o envelhecimento é uma fase da vida que está cada vez mais sendo discutida devido aos avanços na medicina e a preocupação com o cuidado do idoso. Discutiu-se também a questão das doenças que são comuns com o envelhecimento, sendo as que apresentam maior temor entre os idosos e seus familiares, as demências. Dentro dessas, há a mais comum, divulgada e estudada, a Doença de ALZHEIMER, que é um tipo de demência muito comum com o envelhecimento.

Com as estimativas de que nos próximos 40 anos o número de idosos no mundo chegará a dois bilhões de pessoas, políticas públicas, conscientização, orientação e treinamento da família, incentivo as escolas e a educação de cuidadores são fundamentais para que a sociedade envelheça com dignidade, respeito e saúde. Destaca-se que a educação e essa relação entre o profissional da área da saúde, geralmente, um enfermeiro, a família, o idoso deve ser a mais harmônica possível, para que as dificuldades não se tornem problemas de difícil resolução. Assim, o envelhecimento é algo natural que pode ser saudável ou não, que para este caso, o cuidado e o respeito para com o idoso é fundamental, sendo o papel da família importantíssimo neste processo. Inclui-se nesta relação o cuidador, que pode ou não ser um familiar. Este profissional é de indiscutível importância, pois é ele que ficará grande parte do tempo na companhia e realizando os cuidados com o idoso, principalmente, aquele que é portador de alguma demência como a Doença de Alzheimer. Dessa forma, a escolha e o treinamento/educação do cuidador é fundamental para que o idoso envelheça com respeito e a família tenha a certeza que o cuidado está sendo feito com dignidade.

\section{REFERÊNCIAS}

ARAÚJO, V.S.; GUERRA, C.S.; MORAES, M.N.; SILVA, J.B.; MONTEIRO, C.Q.A; DIAS, M.D. Discurso do sujeito coletivo sobre educação em saúde no envelhecimento: estudo descritivo. Online Brazilian Journal of Nursing. v. 12, n. 3, 
2013. Disponível em: <http://www.objnursing.uff.br/index.php/nursing/rt/printer Friendly/4093/html_2.>Acesso em: 18 jan. 2015.

ABRAZ- ASSOCIAÇÃO BRASILEIRA DE ALZHEIMER.; O que é grupo de apoio, 2015. Disponível em: <http://abraz.org.br/gestao-atual/criacao-de-grupos-de-apoio>. Acesso em: 16 dez. 2018

BARROS, A., MARTINS, F., DA CONCEIÇÃO MOURA, M., \& LIMA, E. DEMÊNCIA DE ALZHEIMER: A SOBRECARGA DO CUIDADOR FAMILIAR. Anais Concifa, v. 1, n. 1, 2018.

BERNARDO, L. D.; RAYMUNDO, T. M. Ambiente físico e social no processo de intervenção terapêutico ocupacional para idosos com Doença de Alzheimer e seus cuidadores: uma revisão sistemática da literatura. Cadernos Brasileiros de Terapia Ocupacional, v. 26, n. 2, 2018.

BRASIL. Câmara dos Deputados. Lei n. 10.741, de 1ำ de outubro de 2003. Dispõe sobre o Estatuto do Idoso e dá outras providências. Brasília. 2003. Disponível em: <http://www2.camara.leg.br/legin/fed/lei/2003/lei-10741-1-outubro-2003-497511norma-pl.html>. Acesso em: 18 jan. 2015.

BRASIL. Ministério da Saúde. Portaria no 2528 de outubro de 2006. Aprova a Política Nacional de Saúde da Pessoa Idosa. Brasília. 2006. Disponível em: $<$ http://www.saude.mg.gov.br/index.php?option=com_gmg\&controller=document\&id= 536>. Acesso em: 18 jan. 2015.

BRITO, A. M. M; CAMARGO, B. V; CASTRO, A. Representações sociais de velhice e boa velhice entre idosos e sua rede social. Revista de Psicologia da IMED, v. 9, n. 1, p. 5-21, 2017.

BRUM, A. K. R., CAMACHO, A. C. L. F., VALENTE, G. S. C., SÁ, S. P. C., DA COSTA LINDOLPHO, M., \& DOS SANTOS LOUREDO, D. Programa para cuidadores de idosos com demência: relato de experiência. Revista Brasileira Enfermagem, Brasília, v. 66, n. 4, jul./ago. 2013. Disponível em: <http://www.scielo.br/scielo.php?script=sci_arttext\&pid=S0034-

$71672013000400025 \&$ Ing=en\&nrm=iso >. Acesso em: 18 jan. 2015.

BuRlÁ, C., CAMARANO, A. A., KANSO, S., FERNANDES, D., \& NUNES, R. Panorama prospectivo das demências no Brasil: um enfoque demográfico. Ciência e Saúde Coletiva, v. 18, n. 10, 2012, p. 2949-2956, 2013. Disponível em: <http://www.scielo.br/scielo.php?script=sci_arttext\&pid=S1413-

$81232013001000019>$. Acesso em: 18 jan. 2015.

CAMPOS, C. R. F., DE CARVALHO, T. R., BARHAM, E. J., DE ANDRADE, L. R. F., \& GIANNINI, A. S. Entender e envolver: avaliando dois objetivos de um programa para cuidadores de idosos com Alzheimer. Psico, v. 50, n. 1, p. 29444, 2019.

CAPUCHA, L. Envelhecimento e políticas sociais: novos desafios aos sistemas de protecção: protecção contra o" risco de velhice": que risco?. Sociologia: Revista da Faculdade de Letras da Universidade do Porto, v. 15, 2017. 
CARDOSO, S., DA SILVA, R., CHAVES SÁ, S. P., SABÓIA, V. M., DE OLIVEIRA PADILHA, J. M. F., DE ALMEIDA NOGUEIRA, G., ... \& NUNES MAIA, T. Tecnologia educacional: um instrumento dinamizador do cuidado com idosos. Revista Brasileira de Enfermagem, v. 71, 2018.

CAVALCANTI, I. F. D. M., SILVA, A. C. B., DA SILVA, A. K., DA SILVA GOMES, B. M., FERREIRA, Y. A., \& DE LIMA JUNIOR, A. A. Assistência de enfermagem aos idosos portadores do mal de alzheimer. Revista Saúde-UNG-Ser, v. 11, n. 1 ESP, p. 58, 2018.

CECATO, J., GALEOTE, L.; MARTINELLI, J.E.; Sensibilidade da praxia visuoconstrutiva do moca no Comprometimento Cognitivo Leve e doença de Alzheimer. Neuropsicologia Latinoamericana, v. 10, n. 3, 2018.

CESÁRIO, V. A. C., LEAL, M. C. C., MARQUES, A. P. D. O., \& CLAUDINO, K. A. Estresse e qualidade de vida do cuidador familiar de idoso portador da doença de Alzheimer. Saúde em Debate, v. 41, p. 171-182, 2017.

COOPER, H.M. Integrating Research: a guide for literature reviews. 2. ed. London SAGE publication, [s.I], v.2, p.155, 1989.

GIL, A;C.; Como Elaborar Projetos de Pesquisa. 5 ed. São Paulo: Atlas, 2010.

HEK, G. Systematically searching an reviewing leterature. Nurse researcher. 2000.

JESUS, L., GARCIA, A., CARPES, F. P., CARPES, P. B. M., \& SCHIMIDT, H. L. Neuroproteção do exercício físico de força versus exercício físico de corrida na doença de alzheimer. Anais do Salão Internacional de Ensino, Pesquisa e Extensão, v. 10, n. 2, 2019.

JERÔNIMO, G. M. Produção de narrativas orais no envelhecimento sadio, no comprometimento cognitivo leve e na doença de Alzheimer e sua relação com construtos cognitivos e escolaridade. 2016. $201 \mathrm{f}$. Tese (Doutorado em Linguística) - Pontifícia Universidade Católica do Rio Grande do Sul, Porto Alegre, 2016.

JESKE, T. G., BLASI, T. C., FILIPPIN, N. T., DE OLIVEIRA, F. S., ILHA, S., ZAMBERLAN, C., ... \& DOTTO, P. P. Grupo de apoio aos familiares/cuidadores de pessoas com a doença de Alzheimer. Disciplinarum Scientia| Saúde, v. 19, n. 1, p. $1-10,2018$.

DIAS JÚNIOR, C. S.; COSTA, C. S. O envelhecimento da população brasileira: uma análise de conteúdo das páginas da REBEP. Anais, p. 1-21, 2016.

MANCINI, M.C. Estudos de revisão sistemática: um guia para síntese criteriosa da evidência científica, 2007.

MEDEIROS, D. V., SANTOS, W. N. D., SOUSA, M. D. G. D. M., SILVA, T. D. C. D. D., 
SILVA, P. T. P., \& CASTRO, S. D. F. F. D. A percepção do idoso sobre a velhice. Revista de enfermagem. UFPE on line, p. 3851-3859, 2016.

MIRANDA, G. M. D; MENDES, A. da C. G; DA SILVA, A. L. A. O envelhecimento populacional brasileiro: desafios e consequências sociais atuais e futuras. Revista Brasileira de Geriatria e Gerontologia, v. 19, n. 3, p. 507-519, 2016.

MORAES, EDGAR NUNES DE; MARINO, MARÍLIA CAMPOS DE ABREU; SANTOS, RODRIGO RIBEIRO. Principais síndromes geriátricas. Revista de Medicina Minas Gerais, v. 20, n. 1, p. 54-66, 2010.

MOREIRA, S. V; JUSTI, F. R. dos R; MOREIRA, M. Intervenção musical pode melhorar a memória em pacientes com doença de Alzheimer? Uma Revisão Sistemática. Dementia \& Neuropsychologia, v. 12, n. 2, p. 133-142, 2018.

NASCIMENTO, A., SANTOS, G., CALDAS, G., \& LOPES, G. Os desafios de ser cuidador de idosos: uma revisão integrativa. Anais Concifa, v. 1, n. 1, 2018.

OLIVEIRA D.C.; D'ELBOUX, M.J. Estudos nacionais sobre cuidadores de idosos: revisão integrativa. Revista Brasileira de Enfermagem ., v. 65, n. 5, p. 829-838, 2012. Disponível em: <http://www.scielo.br/pdf/reben/v65n5/17.pdf>. Acesso em: 18 jan. 2015.

OLIVEIRA, R. W., FREIRE, R. A., DE JESUS, L. M. A., DOMINGOS, J. N., CAVALCANTI, E. M. G., \& BARROS, A. M. M. S. O Enfermeiro na Relação Cuidador e Idoso Portador de Alzheimer. Semana de Pesquisa da Universidade TiradentesSEMPESq, n. 18, 2018.

PEREIRA C.D.F.D.; PINTO, D.P. S.R.; TOURINHO, F.S.V.; SANTOS, V.E.P. Tecnologias em enfermagem e o impacto na prática assistencial. Revista Brasileira de Inovação Tecnológica em Saúde, v. 2, n. 4, p. 29-37, 2012. Disponível em: <http://www.periodicos.ufrn.br/reb/article/view/3331/2727>. Acesso em: 18 jan. 2015.

PERISSÉ, A. R. S. Revisões sistemáticas e diretrizes clínicas. Rio de Janeiro: Reichmann e Afonso, 2001.REIS, C; BARBOSA, L. M. de L. H; PIMENTEL, V. P. O desafio do envelhecimento populacional na perspectiva sistêmica da saúde. 2016.

SALES, I. M. de. Efeitos do exercício físico aeróbio em idosos com Doença de Alzheimer. 2018. Trabalho de Conclusão de Curso.

SEIMA M. A.; LEONARDT M. H.; CALDAS C. Relação no cuidado entre o cuidaor familiare o idoso com Alzheimer. Revista Brasileira de Enfermagem, Brasília, v. 67, n. 2, p. 234-238, mar./abr. 2014

SENNA DA CRUZ, L. Avanços e dificuldades na saúde do idoso: desafios para o enfermeiro no cenário brasileiro atual. 2017. Trabalho de Conclusão de Curso (Curso de Enfermagem) da Instituição Anhanguera Educacional II. 2017

SIQUEIRA, J. F., ANTUNES, M. D., DO NASCIMENTO JÚNIOR, J. R. A., \& OLIVEIRA, D. V. Efeitos da prática de exercício de dupla tarefa em idosos com 
Doença de Alzheimer: revisão sistemática. Saúde e Pesquisa, v. 12, n. 1, p. 197202, 2019.

TEIXEIRA, E; MOTA,V;M;S;S. Educação em Saúde. Tecnologias educacionais em foco. São Caetano do Sul: Difusão Editora, 2011.

VERAS, R. Envelhecimento populacional contemporâneo: demandas, desafios e inovações. Revista Saúde Pública , v. 43, n. 3, p. 548-554, maio./jun. 2009. 